\title{
Some Important Problems and Progress in Micro / Nano- scale Thermal Science and Engineering
}

\author{
Yuanming Zhao,Anna Qiu,Juexian Qi \\ Engineering College, Chizhou Jiaotong University, Anhui, China
}

\begin{abstract}
This paper describes the significance, content, progress and corresponding basic theory and experimental research methods of micron/nanometer scale thermal science and engineering, which is one of the latest cutting-edge disciplines, and analyzes the effects of micron nanometer devices on the scale effect series of challenging hot issues, discussed the corresponding emergence of some new phenomena and new concepts, pointed out that the micron/nano thermal science aspects of the recent development of several types of theory and experimental technology success and shortcomings, and summed up a number for the exploration of the new ways and new directions, especially on some typical micron/nano-thermal devices and micro-scale biological heat transfer in some important scientific issues and their engineering applications were introduced.
\end{abstract}

Key words:thermal science, MEMS, nanotechnology, devices, biotechnology

\section{$1 \mathrm{Micron} /$ nano scale thermal science and engineering development}

The nature of the material is shown in Figure 1 [1]. Its size covers from the nano $(10-9 \mathrm{~m})$ to the light of such a very broad range of the past is the most studied the object of human senses, science in recent years and an important trend in technological progress is toward the development of miniaturization, and people's attention is gradually shifting from macroscopic objects to phenomena that occur in small scale and / or rapid processes and their corresponding devices (see Figure 2) [2, 3 ], where the micro-electromechanical system (MEMS) has achieved great success and is rapidly expanding into a variety of industrial processes, such as electronic and mechanical components in one, generally using integrated circuit processing method as shown in Fig. 1, the size of the micro-electromechanical system is four orders of magnitude larger than the diameter of the hydrogen atom, but four orders of magnitude smaller than the size of the conventional manmade instrument [1], and the nano-device further advances the microelectromechanical system miniaturization due to the continuous progress of modern manufacturing and application technology, 'micromechanical' or 'satisfied machinery' size is at an unusual rate of decline, while its performance has been maintained even better, so far, some real molecular level machines such as rotors, gears, switches, gates, grille, motors and other production has even become possible [4]. Because of this, almost all engaged in the people in the field of research believe that a revolution is coming quietly. [5] Over the past two to three decades, people have invested a great deal of time and money in finding excellent ways to create fully sophisticated and sophisticated electronic systems. Such as instruments, medical systems, biosystems, robots, design, navigation and computer applications. Many of the world's leading universities incorporate MEMS and its disciplines into their teaching and research projects. Countries and regions such as the United States, Japan, Sweden, Germany, Singapore and China Taiwan, and other important projects are microelectromechanical systems development and related research to give strong support, such as in the United States, early support microelectromechanical systems research of the funding comes mainly from the National Foundation, about \$ 1 million per year, after the US Department of Defense also Into the support of the ranks of its funding level and each project and even up to 50 million US dollars. [5]. China has also put a lot of manpower and material resources earlier to carry out this discipline research, and in the nano-science of some areas such as directional carbon nanotube arrays and one-dimensional nanowires, have also achieved remarkable results, all of which illustrate the unique charm and significance of the nanometer nanoelectromechanical system itself.

People often attribute the origins of MEMS and nanotechnology to the Nobel Prize winner, Richard Feynman, in a classic paper 'There' at the American Conference on Physics at the West Coast Conference in 1959 'There' plenty of room at the paper 'was published in full text after a lapse of 33 years. Later, another early paper,' Infinitesimal machinery 'by Dr. Feynman, was also re-published in the same publication [7]. These two articles scientifically foresee many of the important features of nanometer nano-materials: 'nano-technology' refers to the use of new technologies such as laser beam, ion beam and electron beam grinding to complete the ultra-fine material processing technology, which can be defined as '0.1-100 nm (from atomic size to light wavelength) scale of the key role of the technology.' This meaning is of course very wide, it can include such as ultra-fine powder processing, electronic and X-ray etching and film production. Micromachining is also a new engineering field defined by the above definition, which is the so-called 'three-dimensional engraving of silicon materials using standard semiconductor processing technology the use of such technology, people have made a lot of very small mechanical devices, such as the size of much less than $1 \mathrm{~mm}$ pressure, flow sensors and accelerometers, including a similar size of the heat exchanger, air turbine, micro-combustion chamber and electronic motors, as well as a set of gas in the production of a set of gas offset copy. Microelectronics and molecular biology is another two areas of emphasis on miniaturization [3], some of the recent outstanding examples such as bio-chip, bioreactors and microelectronic memories. It is not difficult to understand that the common feature of all microscale science is that the transport of matter and energy takes place within a limited tiny structure, and the transport and interaction of matter necessarily involves the conversion of flow and/ or energy. In addition, any molecular remodeling in the chemical reaction or phase transition process necessarily involves the energy exchange problem with the surrounding environment. Therefore, all micro-system design and application, a comprehensive understanding of the system in a specific scale within the micro-electromechanical properties and thermal properties of materials, thermal behavior has become however, the current scientific and engineering level is still unable to do this step [3,5], so, a new branch of modern thermal science - micron/ nano-scale thermal science came into being. With other areas within 
the micro as the scale of engineering is flourishing, micron / nanometer scale heat transfer engineering is becoming one of the most exciting disciplines in thermal science. Kotake [9] has proposed the introduction of a new discipline 'molecular mechanical engineering' science and macro continuous media engineering (mechanical engineering) between bridges.

Early micro-scale heat transfer studies focused on thermal problems, and then extended to radiation and convection problems. The micro-scale thermal conductivity depends on the thickness of the material can be traced back to the 20th century, 30 years, and the first by the physical In the late 1960s, thermophysicists, represented by Professor Tian Changlin at the University of California, Berkeley, began to notice the scale effect of heat transfer problems in a series of engineering devices, heat transfer is quietly rising, especially in the late 1980 s to progress more quickly. All this is closely related with the practical application, for example, due to the rapid development of low-temperature technology and thin metal film and metal wire application growth needs, Tien et al [ 10] on the thin metal film and metal lines were calculated and found that its conductivity and thermal conductivity are lower than the corresponding value in the macroscopic case, one of the reasons is close to the surface of the average free electrons due to the interface of the scattering will be shortened, and the electron mean free path increases with the decrease of temperature, especially at low temperature, when the average free path of energy carrier (such as electron) is the smallest of the given sample In the order of magnitude, the transport of these carriers will reflect the dependence on the size of the sample [11], resulting in the traditional theory of the predicted physical properties of the sample and its real value there is a significant deviation, it is these theories and experiments the observed contradictions contribute to the development of micro-scale thermal science. Micro-scale heat transfer and fluid science cover a very broad field [12-20], such as solid-liquid films, semiconductor devices, optical devices, superconducting devices, chip cooling devices, micro-electromechanical systems, biochips, microsensors, laser processing, thermal medical engineering, life and thermal science and contains a lot of challenging topics.

In order to give the reader a specific understanding of micron/ nano-scale thermal science and engineering research, some typical examples are briefly presented and the important scientific questions are analyzed and summarized.

\section{Some typical micro-scale heat transfer engineering problems}

\subsection{Heat transfer in films}

In 1987, the Swiss scientists found that $\mathrm{YBa} 2 \mathrm{Cu} 3 \mathrm{O} 7$ ceramics in the $35 \mathrm{~K}$ above the superconductivity that is high-temperature superconductivity. This discovery suddenly aroused unprecedented worldwide research boom. For the first time that the existence of a superconductor and semiconductor can be the temperature range of the work, so a set of superconductor - semiconductor in a powerful composite device came into being. The basic unit of such devices is a deposition of silicon or gallium arsenide substrate on the high temperature superconducting film [21], and its internal and external heat transfer problems are closely related to the research and application of superconductivity, so the study of the thermophysical properties of the film and its heat transport is naturally the key to improve the performance of the instrument. A series of studies [21 -23], for a certain temperature and thickness of the high temperature superconducting film or dielectric film, by the interface caused by the energy carrier of the average free path changes, will lead to thin film heat transfer law deviation from the Fourier theorem of the situation. In this respect, the Boltzmann equation is recognized as a highly universal and effective tool. Majumdar [22] thus developed a phonon radiator the study shows that the thermal conductivity of lattice vibrations or phonons is in the form of radiation heat transfer in the micro-scale region, and a large number of studies have been carried out by the Boltzmann theory. Deepened the understanding of the film heat transfer law.

\subsection{Heat transfer in particles and point structures}

Two typical examples of this aspect are monocrystals and polycrystals. [24] When the unit cells of the crystal are repeated in the three-dimensional space and form a macroscopic object without any periodic discontinuities or interfaces. However, the solid is usually in the form of a polycrystalline, which consists of a plurality of single crystals called particles, and the individual clusters are separated by an interface called a particle boundary, the interface to a solid, chemical, electrical and thermal properties, crystal defects (such as point defects and dislocations) also strongly affect the nature of the solid except for the grain boundary. In addition to crystalline solids, there are also known as amorphous or the glassy solid, although it is indeed ordered in a short lattice or even lattice spacing, does not have long-range orderliness. Obviously, similar to the heat transfer problem in the film, when the particles and the point structure when the size is small to a certain extent, the classical thermal science theory applicable to macroscopic objects is no longer effective. It has been paid more and more attention in recent years to the measurement of thermophysical properties of particles and point structures.

\subsection{Heat conduction in narrow lines and quantum wells}

The research on the quantum well structure based on gallium arsenide has been progressing rapidly in recent years [25]. The quantum well structure is usually composed of one or more layers of ultra-low ( $\sim 200$ or less) low energy gap embedded in high energy gap media semiconductor layer composition. It is found that the thermal conductivity is smaller than the corresponding macroscopic volume value even at room temperature [25]. The relatively narrow metal wire (such as nanowire) is another thermophysical depending on the size (such as diameter). In addition, it should be noted that the micron / nanometer scale of the heat problem in the importance of nanotechnology is increasingly apparent, such as Schwab et al [26] found that to try to create in the number the problem of thermal confinement over a quantum wire or device within a molecular thickness dimension is due to the fact that heat is only transmitted through a limited channel in a very small scale structure, so they often at present, the research and application of such problems have just started, but has attracted the attention of scholars in many fields [27].

\subsection{Computer components and their heat transfer problems}

In recent years, a significant feature of the development of the microelectronics industry is the expansion of personal computers and workstations, where, in these small or medium-sized systems, without exception, forced convection air is used to cool the heating device. The relationship between the heat dissipation at the system level and the volume of the system is shown [28]. It can be seen that all the data falls within the band between the two parallel lines, and the slope of the band can be seen, the volume dissipation density in the system is almost independent of the system size and ranges from about 3000 to $7000 \mathrm{~W} / \mathrm{m} 3$, where the volume of heat dissipation in the notebook is the highest, up to $7000 \mathrm{~W} / \mathrm{m} 3$, and the high density of heat difficulty of cooling the tiny system is that [28]: first, the cooling air rate cannot be too high to minimize noise; second, only a small cooling fluid space 
is allowed; and thirdly, fourth, the low cost requires the use of plastic packaging chip as much as possible, which will increase the thermal resistance between the chip and the surface of the module, so the heat will be the main set on the substrate. So, look for a highly efficient method of heat transfer performance of heat transport has been the subject of people to explore.

\subsection{Micro heat exchangers and heat transfer problems}

Micro heat exchangers have a wide range of applications, and have been fully used in electronic devices, micron/nano electromechanical systems, some of the most advanced biotechnology and micro-medical instruments. With the current micro-system and nanotechnology rapid development, a variety of refreshing micro-heat exchanger is still endless. The development of modern micromanufacturing technology has made the processing by a number of hydrodynamic diameter between $10-103 \mu \mathrm{m}$ micro-pipe composition of the heat exchanger possible [29] such flow channels or staggered fins are usually made on silicon, metal or other suitable material sheets, each of which may be a separate plate heat exchanger, or may be stacked and welded together to form a parallel Flow or countercurrent heat exchangers (Figure 4). Channel and fins can be photolithographically or by means of a micro-tool made by precision cutting on a sheet. In addition to the micro-channel heat exchanger, the micro heat pipe is another micro heat exchangers with high heat transport capacity [30], the existence of many complex micro-scale flow, heat transfer and phase transition mechanism to be revealed.

\subsection{Micro gas turbine combustion technology}

One of the most challenging attempts in micro-fabrication technology is the production of micro-turbines, which, despite its small size, still include micro-electric compressors, micro-turbine generators, micro-gas turbines, micro-chillers and micro rocket launchers. Wazitz et al. [31] have recently developed a combustion chamber for micro-gas turbines (see Figure 5). It is justified to develop the motives of such tiny machines, for example, if a successful design generating 10 to $100 \mathrm{~W}$ of electricity and a volume of only $1 \mathrm{~cm} 3$ and less than $7 \mathrm{~g}$ of the micro-gas turbine generator consumed per hour, it will have 10 times the energy density that the best available battery can provide. The difference in design of the conventional device is mostly due to the size reduction, but it is also affected by the relative size of the combustion chamber and the engine, the cycle pressure ratio and the material temperature limit. Some of the special characteristics of the micro-combustion chamber are [31]: (1) the additional energy loss is mainly determined by the higher specific surface area; (2) the use of refractory structural ceramics; (3) microelectromechanical technology only in a number of additional energy loss refractory structure of pottery such as silicon nitride (Si3N4) and silicon carbide ( $\mathrm{SiC}$ ), which have excellent mechanical, thermal and chemical properties to suit the harsh environment. The combustion process in the micro-gas turbine Involving complex chemical reactions, micro-heat transfer, mass transfer and multiphase flow problems, but also includes almost all large-scale burner problems such as high temperature cooling, flame stability, ignition, fuel delivery and plugging. As the micro-size burner itself is greatly reduced, which in turn is significantly different from the macroscopic laws burner.

\subsection{Micro-scale thermal drive technology}

In some cases, the thermal signal is considered to be one of the most suitable tools for controlling some 'tiny' machines [4], and Figure 6 shows the use of micro-bubbles as a driving component designed by Lin et al. [32]. Compared with the thermal drive, some of the traditional micro-actuation techniques such as stack-type piezoelectric actuators to achieve a larger force output, the displacement is relatively small $(<10 \mu \mathrm{m})$; and double piezoelectric chip can get a large displacement, but the output force is relatively small. In addition, the electric drive is very sensitive to particles and humid environment; and magnetically actuated and difficult to process to sub-millimeter scale. It is reported that the use of thermal phase-driven effector can be relatively large force, and can achieve tens of micron displacement [33]. However, also because the micro-heat controller is more sensitive to temperature, they cannot be used in a very high temperature changes. Recently found that, in addition to the electric field, temperature or temperature gradient can play a guiding role in the microfluidic flow on a certain forming surface [34], which may have important application prospects.

Another example of micro-scale thermal control can be found in the printer industry. In the development of micro-electromechanical systems, thermal inkjet print heads are one of the more successful business models based on their sales price and total number. The head (or pen) is enclosed in a replaceable droplet emitter chamber in a volume of 9-50 $\mathrm{cm} 3$, which is typically made of an ink pool and a micro-heating resistance corresponding to the ink jet valve array on the silicon substrate array structure [5]. Figure 7 is a cross-section of a thermal inkjet head with an integrated motion electronic device. Since the quality of the printed image strongly depends on the weight of the ink droplets, the control technique for achieving droplet emission is once Figure 8 shows the ink droplets in a class of ink droplets with the decline in the weight of the situation, the horizontal line represents the human eye can perceive the minimum droplet weight, below this threshold, you can get high print the image with precision.

\subsection{Micro-scale biothermal heat transfer}

The life process occurs on three spatial scales [35], the nano-scale corresponding to the behavior of organic molecules, the micron scale associated with the behavior of individual cells in the tissue, and the macroscopic scale associated with the behavior of the whole organism. The effect of temperature on the life system (cell) and micron (cell) scale. A variety of cell sizes are roughly distributed in several microns (such as sperm and red blood cells) to hundreds of microns (such as oocytes) between the majority of dozens of microns cells is an organism whose outer part consists of a lipid bilayer membrane, which is a solution of the cytoplasm and a number of organelles in the cytoplasm. Studies on the mass transfer problem in the cell scale range have gradually become biological in the study of a focus, the engineering background can be found in a large number of biological materials preserved, freeze-dried, frozen surgery, hyperthermia hyperthermia.

The process of biological heat transfer at micro-scales is virtually all, and in the individual of life, the natural structure of some organisms contains unique micro-mass and energy transport mechanisms. In a recent study, Miaoulis et al. [36] said that it is recognized that biofilms such as insects, plants, lizards and birds have important life significance, and the thickness of each layer on its surface can be $1 \mu \mathrm{m}$ or less, and the organism may have up to 10 layers of biofilm and the air gap alternately composed of layered film, these multi-layer structure evolution so far, can be $0.5-2.0 \mu \mathrm{m}$ wavelength of sunlight interference occurs due to micro-scale radiation effect, the film thickness of the small changes will cause its reflection and absorption the performance of the sun has changed dramatically, and by virtue of the selective evolution of the film thickness and structure, nature has found an excellent application for these multilayer structures, one of which involves biological heat transfer, such as butterfly, its unique film the structure will lead to a 
bright rainbow color, which helps its signal to send, courtship, camouflage and display (as shown in Figure 9), and those not reflected light is used for its own heat changing the film thickness and induce different spectral reflection characteristics.

Micro-scale heat transfer has also been used in some of the most advanced biotechnologies, such as the use of biological tissue in the chemical reaction rate of high sensitivity to temperature, people designed a unique high-performance thermal performance of silicon microstructure, which makes the past It takes about 3 hours of polymerase chain reaction (a well-known biochemical reaction for the expansion of DNA fragments, the author of the idea Mullis won the 1993 Nobel Prize in Chemistry) now only about 20min to complete [37], Which is due to the fact that a reaction volume with a suitable aspect ratio and a large surface is used in the silicon microstructures so that fast heating and cooling rates can be obtained in a low heat quality exchanger in addition to the significant advantages of speed, since the biochemical reaction is usually very demanding on the specificity, rapid heating and cooling also provide additional advantages. With such biochemical reaction device similar to the bio-chip manufacturing methods and its micro-heat transfer mass, micro control, collection and other micro signal will also become a new round of research focus.

\subsection{Molecular machines}

The motive force of science always leads people to discover unknown phenomena and natural laws, and the principles of engineering are driving people to continually challenge newer, more difficult and more efficient systems and devices, both of which are always pavilions and synergistic the pursuit of mechanical processing at the molecular level leads to the creation of a series of corresponding devices, such as: rotor, gear, switch, gate, grille, motor and ratchet, where the molecular motor is compared in the biological system In general, its production has recently become a reality in the laboratory [4]. Koumura et al [4] found that the use of ultraviolet light or by changing the system temperature to stimulate the four separate isomerization steps can be achieved repetitive, single the direction of $360^{\circ}$ rotation around the movement with the microscopic particles of thermal disturbances and reflect the random Brownian movement is similar to the molecular scale of the machine must be very sensitive to its thermal environment, obviously, the thermal control of such devices the research is no longer a distant dream.

\section{Micro devices heat transfer problems arise scale effect and challenging topics}

The study of thermal science and engineering problems in micro devices has led to a series of unexpected and challenging issues that have brought new shocks to traditional ideas, and now it is increasingly recognized that as the scale becomes more and more smaller, the heat and fluid behavior in the device will drastically deviate from the laws described in classical heat transfer and hydrodynamic theory, that is, the thermal fluid behavior in the micron/ nanometer scale region will reflect a strong scale effect, and those that are widely used in the physical quantities such as temperature, pressure, internal energy, entropy, enthalpy and even thermal properties such as thermal conductivity, specific heat, viscosity and so on in the continuous medium system need to be re-understood and given new meanings to these scientific problems. In-depth understanding has become the key to the development of new materials and improve the performance of new micro-devices. For micro-engineering systems, through the micro and mesoscopic approach to establish a basic understanding of the material heat transfer and description. Although the past has been on the various energy transport to explore, but its application to the micro-scale engineering system requires a new field of vision, which is why the micro-scale thermal science s reason.

At present, the researchers try to establish some criteria to determine the effect of heat transfer scale, for example, for the thermal problem, Flik et al. [38] given the regional map pointed out that the average free path for the heat carrier play a decisive role, the layer thickness is about 7 times smaller than the average heat conduction in the thickness direction of the layer, or the layer thickness is about four times that of the average heat conduction along the direction of the layer, it is considered that there is a scale effect in the heat transfer in the layer. But also the new theory itself is very imperfect, and the material scale is very small when the thermal physics laws depend on the fact that many factors also increase the interpretation of the experimental results. In addition, the theory of thermal radiation and thermal convection is also put forward the corresponding scale criterion. The thermal conductivity values ??of the films measured so far vary widely [39] because some of the unique properties of the film (such as film thickness, film-substrate temperature and film deposition) affect thermal conductivity and the uncertainty involved is difficult to estimate accurately. For example, although many theoretical and experimental studies have concluded that the thermal conductivity of the film material is less than one or more orders of magnitude than the material value, it is also found that, the thermal conductivity in the nickel coating material is only slightly smaller than that of the body material. In addition, Volklein and Kessler [40] found that the thermal conductivity of the bismuth metal film (20-400 nm thickness) increases with temperature and the temperature is greater than $300 \mathrm{~K}$, it increases with increasing temperature. In fact, the physical and chemical properties of the thin film material strongly affect the degree of deviation between the thermal properties and the corresponding body material values even if the chemical properties of the film are similar, the microstructure of the film can vary greatly depending on the deposition method. In the optical element, the microstructure, holes and cracks of the terminal material can seriously affect the service life of the device. Laser-induced damage to the parameters of the analysis of these issues need to understand the material at the molecular and atomic level of the structure of its transport characteristics of the impact. To the macro level to explain these issues, a comprehensive and integrated film thermal conductivity model should take into account The anisotropy of the material, the nonuniformity of the material, and the temperature dependence of the membrane material. [41] In view of the differences in the results of many experiments, Tien and Chen [20] put forward a valuable idea, that is, the different laboratories that are engaged in thin film thermal conductivity measurements should be tested against the same batch of samples provided by the same supplier to assess the accuracy and reliability of the methods used and to have a true characteristic of the thermal conductivity of the film complete understanding. Correspondingly, the flow and radiation problem is also necessary to adopt similar research strategy.

\section{Some basic theoretical and experimental research methods of heat transfer for scale micron/ nano}

The study of heat transfer and fluid transport properties can proceed from a macro or micro-approach [24]. The macroscopic approach relies primarily on phenomenological models, without knowing the mechanism of energy transport and the microstructure of the material, which is primarily used to analyze macroscopic scale heat phenomenon, simply know the constitutive relationship or simple 
transport law can be, and thus seem relatively simple, but this method in the understanding and resolution of micro-engineering system involved in the problem and cannot provide enough information, because these systems often need to understand the microscopic mechanism of heat transfer. To date, in theory and computational research, according to the characteristics from the continuum phenomenon to the quantum phenomenon, the researchers proposed a series of quantum molecular dynamics to continuum model method [2, 9]. For the macroscopic continuum phenomenon, the macroscopic variables involved in the system can be obtained by using the traditional continuous momentum, momentum and energy equation. In addition, for some traditional fluid mechanics, heat transfer theory and its corresponding basic equations and interface conditions, physical properties such as moderate correction, but also to achieve the purpose of analyzing some of the micro-system thermal problems. Molecular dynamics equation the molecular characteristics of the physical phenomena which are not obvious in the quantum mechanics effect are revealed, and they also provide knowledge of molecular collision dynamics for molecular statistical theory such as the Boltzmann equation and the direct Monte Carlo simulation method. Physical processes such as the interaction of light and matter and the heat transfer in metal materials, should be analyzed by means of quantum molecular dynamics and by simultaneously solving the molecular dynamics equation and the Schr?dinger equation. In the process of micron/ nano-scale heat transfer theory, two basic problems are necessarily involved. First, any calculation is applicable only to a certain length and time scale range, and in other areas it will fail or cause difficulties. For example, The dynamics simulations are mainly used to study phenomena occurring within 1 to $100 \mathrm{~nm}$, but for a larger or longer range, the method is computationally at least not realistic at the moment, but fortunately, many physical phenomena and Although the engineering problem is in the macro or 'people' level reflected, but in the final analysis is starting from the molecular scale. Currently, There are some difficulties in establishing a model that spans a certain length and time range, and the lattice Boltzmann method is attempting to connect the gap, but further work is needed because the engineering system is often seen by the presence of some mesoscopic phenomena The second problem is how to calculate the computational method, especially at the molecular level, for example, the molecular cluster structure such as C60 can be simulated by molecular dynamics, but the difficulty lies in how to actually investigate the dynamics at the molecular scale transport phenomena, all of which are placed in front of people's problems.

Another very important area is the development of experimental methods for studying micro-scale heat transfer [19]. Several new experimental techniques and measurement methods have been proposed, some of which can be used to obtain the physical picture of micro-scale energy transport phenomena, while other methods advance toward the respective spatial, temporal or energy resolution limits, and in this respect, the beam reflection measurement technique has achieved some success, but its experimental process is highly susceptible to various disturbances. Using atomic force microscopy to electron system and device thermal imaging is another very promising technology. One of the mechanisms for improving this particular method is the use of more accurate temperature measurement devices to replace thermocouple probes. In this respect, semiconductor diodes, resistance thermometers or quartz crystals Figure 2 shows the length of the range from $1-100 \mathrm{~nm}$, and the corresponding time scale from $1 \mathrm{fs}$ $1 \mathrm{~ns}$, these nano-length is the range of scanning probe microscope can range, and thus scan the probe The microscope has been used to study the transmission of charge, photon and heat, but it does not yet have enough time to respond; on the one hand, the nanosecond time scale is equivalent to the ultra-short laser pulse, so picosecond and femtosecond lasers can be used to study the ultrafast kinetic process in photon interaction, but the ultra-short pulsed laser does not have enough spatial resolution. The instruments that combine these extreme space and time limit scales are near-field scanning optical microscopes [2]. These methods are almost the only way to isolate the interaction between electrons and phonons in solids. Now ultrafast lasers have been widely applied to physics and chemistry, and its new engineering applications are constantly expanding, and it has been possible to obtain a skew in the range of $1 \mathrm{fm}$ of the micromechanical structure, which makes the physical and chemical sensory level unparalleled. Note that $1 \mathrm{fm}$ is greater than a chemical bond length small $1 \%$, this detection ability to obtain a fundamental understanding of the behavior of the material in history such examples are not uncommon, that is, once the limits of a measurement method is broken, the new scientific discovery and engineering revolution is coming soon.

\section{Summary and Outlook}

Micron/ nanoscience has become one of the most popular top disciplines in the world. The cornerstone of the 21 st century world economy will be largely based on tiny devices, while micron/ nanoscale thermal science is micro-scale science (Such as thermal physics, thermodynamics, fluid mechanics and thermal testing techniques), physical (cohesive), physical and chemical physical (microfabrication), materials (new material preparation, measurement, thermal evaluation), chemical (microfluidic, micro-fluid, microelectronics and micro-electromechanical systems) reactions, biomedical engineering (micro-pumps, micro-sensors and micromedical instruments), instrumentation, biological information and control (bio-chip, petri dish) and many other areas of a new growth point. Although this article is only a brief introduction to the heat science in the micron/ nano system technology in some important applications, but it has been clear enough to show that, together with other disciplines, the role of thermal science is also irreplaceable the rapid development of micron/ nanometer technology is now under the mutual cooperation and synergistic development of various micron / nano-scale branches. Today, the commercialization of microfluidic and heat transfer devices is in its early stages of development and is currently available of the limited number of devices, micro-scale device volume and weight reduction is contributing to some new engineering applications, it is possible (or already) to open up new markets, and for the basic exploration to provide a new means of research.

In the past few decades, the discovery of new heat transfer in microsystems requires a series of new research methods [19], while the development of the semiconductor industry will further advance the application to the low temperature region, at which point the micro- as in the previous theory, there are still inconsistent and even contradictory results in various theoretical and experimental results, so that in addition to the existing theory to be corrected (such as considering the heat conduction of the to the heterosexual, nonuniform convection problems in the fluid compression, changes in the physical properties of the media, field effects, boundary effects and the particularity of the material structure), in particular, to develop new theories and testing techniques, and to develop new high performance, low-cost micron / nano-thermal devices, and the expansion of MEMS thermal science applications. With basic research is the need for better design tools to make microfluidic and thermal systems into a complete simulation, this type system should include design and process modeling as well as numerical simulations of device fluid dynamics, 
thermal behavior, structural deformation and its performance. People not only ask in the case of condensed matter physicists have studied the problem of micro-scale energy transport over the past few decades, what is the focus of micro-scale thermal science? There is a consensus that core issues should focus on complex materials and engineering systems In this system, the low-temperature and ideal crystal assumptions are no longer established, in which the energy transport within the micro-scale interacts with the surrounding environment, and leads to the ideal that has been studied in the past the unique behavior of the system is not easy to occur within the control system, the mother of the question, micron/ nano-scale thermal science research areas are constantly expanding its life sciences as a clue, it is full of hope, the researches of micron / nano-scale scientific area are trying their best to improve and overcome the limits, and the most exciting parts are the relationship between those equipment, calculations and experiments.

\section{REFERENCES}

[1] Gad-el-Hak M.ASME Journal of Fluids, 1999, 121:5

[2] Tien C L, et al. Microscale Thermophysical Engineering, 1997, $1: 71$

[3] Tien C L, Majumdar A, Gerner F M. Microscale Energy Transport. New York: Taylor lu0026Francis ,1998

[4] Koumura N, Zi Jlstra RW J, van Delden R A et al. Nature, 1999, 401:152

[5] National Materials Advisory Board. Microelectromechanical Systemsadvanced Materials and Fabrication Methods.Washington D C: National Academy Press, 1997

[6] Feynman R. Journal of Microelectromechanical Systems, 1992, 1:60

[7] Feynman R. Journal of Microelectromechanical Systems, 1993, 2:4

[8] Ciofalo M, Collins M W, Hennessy T R. Med . Eng. Phys, 1996,18 :437

[9] Kotake S. JSME International Journal Series B: Fluids and Thermal Engineering, 1995 , $38: 1$

[10] Tien C L, Armaly B F, Jagannathan P S .Proc. 8th Thermal Conductivity Conference. New York: Plenum Press, $1969.13-19$

[11] Ziman J M. Electrons and Phonons - the Theory of Transport Phenomena in Solids. Oxford: Oxford Press, 1963

[12] Bankoff S G.ASME J. of Heat Transfer, 1994, 116:10

[13] Jr. Wayner P C. In:Tien C L, Majumdar A, Gerner F M eds. Microscale Energy Transport. New York :Taylor Francis , 1998.187-227

[14] Goodson K E, Ju Y S, Asheghi M. In:Tien C L, Majumdar A, Gerner F M eds. Microscale Energy Transport.New York :Taylor Francis, 1998 .229294

[15] Ho C M, Tai Y C.ASME J. Fluids Engineering, 1996, $118: 437$

[16] Gravesen P, Branebjerg J , Jensen O S .J. Micromech. Microeng., 1993 ,
$3: 168$

[17] Elwenspoek M, Lammerink T S, Miyake R et al. J. Micromech. Microeng., 1994, 4 :227

[18] Shoji S, Esashi M. J. Micromech. Microeng ., 1994 , $4: 157$

[19] Duncan A B, Peterson G P. Appl. Mech. Rev., 1994, 47:397

[20] Tien C L, Chen G. ASME J. of Heat Transfer, 1994, 116:799

[21] Flik M I, Tien C L . ASME J.of Heat Transfer, 1990, 112:872

[22] Majumdar A .ASME J. of Heat Transfer, 1993, $115: 7$

[23] Jen C P , Chieng C C.J ournal of Thermophysics and Heat Transfer, $1998,12: 146$

[24] Majumdar A. In:Tien C L, Majumdar A, Gerner F M eds. Microscale Energy Transport. New York :Taylor Francis, 1998.3 -94

[25] Chen G, Tien C L. Journal of Thermophysics and Heat Transfer,1993, 7:311

[26] Schwab K, Henriksen E A, Worlock J M et al. Nature, 2000,404:974

[27] Cho A.Science, 2000, 288:591

[28] NakayamaW. In :Tien C L ed. Annual Review of Heat Transfer,1997 .8:1

[29] Weisberg A, Bau H H, Zemel J N. Int. J. Heat Mass Transfer, 1992 , $35: 2465$

[30] Peterson G P, Swanson LW, Gerner F M. In:TienC L, Majumdar A, Gerner F M eds. Microscale Energy Transport. New York :Taylor Francis , $1998.295-338$

[31] Waitz I A, Gauba G, Tzeng Y S. ASME J. of Fluid Engineering, 1998, 120:109

[32] Lin L, Pisano A P , Lee A P. 6th International Conference on Solid State Sensors and Actuators, Digest of Technical Papers.San Francisco, 1991.1041

[33] Bergstrom P L, Ji J , Liu Y N et al. Journal of Microelect romechanical Systems , 1995, 4:10

[34] Kataoka D E, Troian S M. Nature, 1999, 402 :794

[35] Rubinsky B. In:Tien C L, Majumdar A, Gerner F M eds. Microscale Energy Transport. New York :Taylor Francis, 1998. 339 -368

[36] Miaoulis I N, Tada H, Mann S et al. Advances in Heat and Mass Transfer in Biotechnology , ASME, 1997, HTD-Vol.355 BED-Vol.37 :33

[37] Christel L A, Petersen K, McMillan W et al. ASME Journal of Biomechanical Engineering , 1999, 121:22

[38] Flik M, Choi B I, GoodsonK E. ASME J. of Heat Transfer, 1992, $114: 666$

[39] Mirmira S R, Fletcher L S. Journal of Thermophysics and Heat Transfer, 1998, 12:121

[40] Volklein F, Kessler E. Thin Solid Films, 1986, $142: 169$

[41] Guehther A H, McIver H K. Thin Solid Films, 1988, 163 :203 